Alejandro Crispiani Enríquez

\section{Adolf Loos: contra el proyecto ${ }^{2}$}

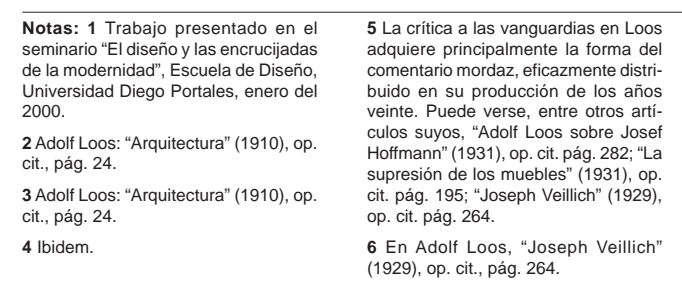
Universi 2 Adtolf pág. 24 3 Adolf Loos: "Arquitectura" (1910), op
cit., pág. 24. 4 lbidem.
8 Massimo Cacciari: "Adolf Loos y su ángel" en Adolf Loos (selección e introducción a cargo de Antonio Pizza), 9 Ibidem. 10 Adolf Loos: "Sobre el ahorro", op. cit.,
Pocas figuras de la arquitectura contemporánea han permanecido tan irreductibles como Adolf Loos a cualquier intento de asimilación a las líneas principales de ésta. Cuando se considera la totalidad de su producción teórica, que no consta propiamente de ningún libro sino de algunos ensayos, en su mayoría célebres pero esporádicos, y de una infinidad de artículos y conferencias aparecidos en los más diversos medios periodísticos, aparece con nitidez hasta qué punto las formulaciones de Loos fueron ajenas y en gran medida contrapuestas, por ejemplo, a los postulados formativos de la arquitectura moderna haciendo incomprensible su papel de precursor. En tal sentido, lo que resalta como más evidente es su frontal rechazo, expresado en comentarios como aguijones, de las intenciones y del mundo de valores de las vanguardias históricas y de algunos de sus principales representantes. Pero aún aquella crítica que, como la Tendenza italiana con Aldo Rossi a la cabeza, ha considerado esta ajenidad de Loos al ideario de la arquitectura moderna y ha hecho de ella, casi podría pensarse, su gran atractivo, no ha podido evitar una cierta incomodidad frente al pensamiento del arquitecto vienés. Incomodidad en gran medida cultivada por él, pero no por ello menos efectiva.

Parte de esta incomodidad, entiendo, se debe al constante desmontaje y anulación de una categoría central para la arquitectura moderna: la idea de proyecto, en todas las acepciones que ésta palabra tiene dentro del campo de la arquitectura. Efectivamente, a lo largo de toda su producción escrita y cualquiera sea su interlocutor, Loos realiza una operación que va a llevar hasta sus últimas consecuencias su intención de concebir a la arquitectura y al diseño moderno extirpando de ellos casi por completo toda noción de proyecto. Esta crítica radical a la idea de proyecto no reconoce en su obra una formulación precisa, no se encuentra desarrollada en su especificidad, sino que su eficacia reside justamente en su punzante dispersión, apareciendo y reapareciendo en sus escritos una y otra vez, desplegándose en múltiples facetas.

\section{C ultura versus proyecto}

La crítica de Loos a la idea de proyecto se inscribe, en principio, dentro de uno de los temas principales de su pensamiento: la arquitectura pertenece a la cultura, es una de sus manifestaciones. Esta afirmación aparentemente inofensiva conlleva distintas consecuencias, pero en principio es necesario preguntarse ¿qué es cultura para Loos?
Entre las diversas definiciones que formula, quizás la más ajustada a su pensamiento sea aquella según la cual cultura sería "aquel equilibrio de la persona interior y exterior, lo único que posibilita un actuar y un pensar razonable" ". Se entiende a la misma, por lo tanto, como la suma de las prácticas, saberes y artefactos creados por el hombre para relacionarse con la realidad de su tiempo y con la naturaleza. Cuando esta relación es armónica, cuando nada la distorsiona y el vínculo entre la realidad interior y la exterior del hombre se realiza con natural fluidez, estaríamos, para Loos, en presencia de una cultura. No todas las épocas, en su opinión, pudieron alcanzar una cultura. Particularmente el siglo XIX, de todas las etapas humanas, no habría logrado conformar este vínculo. El equilibrio entre interior y exterior se habría roto ya que el hombre del siglo XIX permanecería ajeno a su propia producción, desligado de los productos de su época por un sistema de representaciones y de pseudosaberes que se arrastraría de otros momentos históricos y que le impedirían acceder a las fuerzas de la realidad que constituyen su propio tiempo. Fuerzas de la realidad que, por otra parte, no pueden dejar de actuar en una precisa dirección y que determinan en definitiva "lo concreto" que rodea al hombre: los utensilios, los artefactos, las cosas.

Se desprende del pensamiento de Loos que la cultura estribaría fundamentalmente en la capacidad de los integrantes de una sociedad para conectarse natural y razonablemente con lo concreto de su tiempo, y se manifestaría principal-mente en la comprensión de los propios objetos, construcciones y artefactos creados por el hombre para sí con el fin de actuar sobre la realidad, y en definitiva, para comunicarse con el mundo.

Comprender los objetos, saber usar las cosas: ésa sería la máxima expresión de una cultura. Ésa fue una de las grandes tareas que el propio Loos se impuso: enseñar a los vieneses de fin de siglo ("y al mundo") las pequeñas pero infinitas operaciones que les permitirían tener una cultura. Cómo sentarse en un fateuil inglés, cómo salar la comida, qué zapatos elegir, qué muebles comprar para la casa, cómo combinar las prendas de vestir, son las cuestiones que más seriamente se abordan en sus escritos, sin la ironía que suele tener reservada para los grandes temas. Muchos de sus artículos no son otra cosa que fragmentos de un manual de instrucciones y una guía de comportamientos para el hombre moderno. Ahora bien, para Loos, las fuerzas que empujan a la cultura, si bien comprensibles en su lógica de desarrollo y sin duda "razonables", no pueden ser encauzadas por ningún acto de voluntad. La "corriente perfectamente regular" de la cultura de la que habla Loos ${ }^{3}$ es demasiado fuerte como para poder ser modificada decisivamente desde su interior. Se puede prever el desarrollo de la cultura pero ninguna acción voluntariosa podría modificar su rumbo. Justamente, la cultura se desenvuelve "sin mirar hacia delante ni hacia atrás", es en lo sustancial un vínculo con lo presente, con lo concreto, y también con lo contingente. Es por eso que Loos rechaza cualquier noción de "proyecto", entendido éste en términos de acción general, en el campo de la cultura. Aunque nunca se hace explícito, la posibilidad de previsión y de supuesta acción sobre el futuro que supone todo proyecto, sólo implicaría una demora y una traba en su desarrollo.

En este punto es necesario introducir otra premisa del pensamiento de Loos: la cultura no abarca al arte sino que se halla irreconciliablemente separada de él. Aunque resulta bien conocida, es necesario, aunque sólo sea someramente, hacer referencia a esta distinción que establece. El arte, para Loos, sería una fuerza contraria a la cultura, que trataría de instalar en ella determinados valores inexistentes todavía en su seno. Sería una fuerza perturbadora que intentaría sacudir los fundamentos profundamente conservadores de la cultura, introduciendo valores que la misma sólo conseguiría asimilar en el futuro. Esta operación de premonición de valores futuros que sería el arte sólo podría ser producto de un genio, de una individualidad absoluta y aislada de su cultura, y dotada del don de la creación. Siguiendo en gran medida los postulados de la teoría romántica, el genio, para Loos, crea estos nuevos valores sin proponérselo, inocentemente. Como diría Ruskin en relación a Turner, el genio "sólo consigue su meta cuando no se propone ninguna". Vale decir, esta irrupción en el campo de la cultura que el genio provoca no es producto de su voluntad, sino que se le da "naturalmente". El genio no se propone, para Loos, romper con las fuerzas de la cultura, simplemente no puede evitarlo. Crea sin elección e inexorablemente. Y esta creación se dirige al futuro, oponiéndose a las fuerzas vitales de la cultura, que constituyen el presente.

En tal sentido, la arquitectura o, mejor dicho, la actividad del hombre relacionada con la realización de edificios y espacios habitables, pertenece, por su propia naturaleza, a la cultura, no al arte. Como él mismo ha señalado en su famoso ensayo "Arquitectura", sólo una pequeña 
porción del quehacer humano escapa al ámbito de la cultura, aquella que tiene que ver justamente con lo que escapa a la contingencia y a lo concreto de la vida cotidiana: las construcciones que celebran valores que exceden al presente, básicamente la arquitectura funeraria y los monumentos, los hechos físicos en los que encarnan los valores cívicos y religiosos de una sociedad y su idea de la muerte. Como es bien sabido, éstos entrarían para Loos en la esfera del arte, constituyendo el único segmento de la actividad constructiva del hombre del que se podría hablar con propiedad de Arquitectura. El resto es cultura, no arquitectura ${ }^{4}$.

\section{EI proyecto moderno o la apariencia de lo nuevo}

Como parte de la cultura, entonces, todo lo relacionado con la edificación y la producción de objetos de uso no puede ser sometido a un proyecto, por más que éste se presente en nombre de lo moderno. De ahí su rechazo a todos los movimientos modernistas en el ámbito de la arquitectura: en un primer momento a la Secesión y los Talleres Estatales Vieneses, posteriormente, a todas las vanguardias de los años veinte: el purismo francés, la Escuela Bauhaus, la Nueva Objetividad, el Constructivismo ruso ${ }^{5}$. Todas estas corrientes tuvieron entre sus objetivos implantar una nueva forma de hacer y de construir, basada en principios estéticos o técnicos que suponían una fractura con el presente y una proyección hacia el futuro.

Pero para Loos no es posible inventar lo nuevo. Lo nuevo sólo puede irse produciendo dentro de una determinada tradición, dentro de un determinado rumbo marcado de manera natural por la cultura. Nadie debe proponerse crear algo nuevo en lo que a los objetos del hombre se refiere. La novedad surgirá espontáneamente donde las necesidades la llamen a actuar y donde las condiciones de la cultura sean las propicias para aceptar y desarrollar esa novedad dentro de una cadena constante. Un pequeño, infinitesimal cambio en esta cadena, puede tener consecuencias más profundas que cualquier intento, por impresionante que sea, de actuar desde fuera de ella. Como lo señala el mismo Loos: "Es bien sabido que todas las frenéticas elucubraciones sobre la manera de vivir, en cualquier país, no han logrado mover al perro del calor de la estufa, que todo el tráfico de Asociaciones, Escuelas, Profesorados, periódicos y exhibiciones no ban logrado dar a luz nada nuevo" ${ }^{6}$. Lo moderno consiste justamente en saber detectar con precisión dónde y cómo se produce lo nuevo, en esperar que se desarrolle "naturalmente" y según su propia lógica. Por ejemplo, lo verdaderamente nuevo del siglo XIX en relación con el espacio doméstico son los nuevos avances técnicos, la luz eléctrica, los sistemas de cañerías, la calefacción, etc. Ahora bien, sería inútil forzar su imposición inventando una estética a su medida. Su necesidad sería tan potente que no necesitaría de ninguna teorización ni de ningún estímulo artificial, ajeno a su más estricta razón de ser y a sus modos de desenvolvimiento. Lo nuevo no puede ser inventado, su asimi-lación puede hacerse más fácil o más difícil, más lenta o más rápida, pero en realidad el margen es estrecho, su evolución es irreversible e incontenible.

En consecuencia, lo que la cultura ha dejado atrás no podría revivirse, como tampoco sería posible matar prematuramente lo que sabemos que va a morir de todas formas en términos de fenómenos culturales. Tal es el caso, por ejemplo, del ornamento. En su famoso ensayo "Ornamento y delito", escrito en 1908 y publicado en 1913, Loos denuncia al ornamento como una práctica cultural permitida, que ya no estaría "orgánicamente vinculado" a la cultura propia del siglo XIX y que sería, por lo tanto, una pérdida y un despilfarro de trabajo humano, condenando a quienes lo exigieran en cualquier ámbito de la producción material como inmorales. Cuando en los años veinte las vanguardias arquitectónicas asumen esta posición y postulan la supresión radical, programática y voluntaria de todo ornamento, Loos reacciona negativamente contra ellas y en especial contra este intento. Contradiciéndose en algún grado, pero sólo en algún grado, con su posición anterior, sus argumentos contra los puristas modernos son que no es posible eliminar al ornamento por la sola decisión de un grupo de personas, arquitectos o no. Su desaparición tiene que ser tan espontánea como su aparición; agotadas las fuerzas culturales que le dieron origen, y sólo en ese momento, desaparecerá. Es posible exponer su improductividad presente, pero forzar su desaparición es igualmente improductivo. A lo que se opone Loos es que al ornamento, entendido como una intrusión del arte dentro del campo de la cultura que resultaría insoportable para el hombre moderno, se lo combata en nombre de otra operación estética, basada en este caso en la supresión del ornamento, como es la propugnada por la Bauhaus o los puristas franceses ${ }^{7}$. La abstracción moderna no sería, por lo tanto, para Loos, distinta del ornamento histórico. Ambas implican una intromisión de carácter artístico en la vida, ambas atentan contra la cultura. La diferencia es que la segunda, justamente, se hace en nombre de lo moderno y se funda en una idea de proyecto, vale decir, de radical transformación del presente con los ojos puestos en el futuro. Como ya se ha visto, esto supondría, en la lógica de pensamiento de Loos, una contradicción: no se puede ser moderno desentendiéndose del presente y de la cadena de acontecimientos que llevaron hacia él. Para Loos, la idea misma de un "proyecto moderno" resulta inviable; no es posible postular un proyecto, sea cual fuere su envergadura, y ser moderno a la vez. Lo moderno no puede ser impuesto. Existe una tradición, vale decir, una cadena de graduales pero decisivos progresos y existe una situación moderna. De la adecuada y natural simbiosis entre ellas surgirá la arquitectura como parte de una cultura. No hay posibilidad de proyecto.

En tal sentido, resulta claro que, en el pensamiento de Loos, esta tentativa de un "Proyecto Moderno", no sería más que una de las tantas formas de "falsa modernidad" que habrían emergido frente a la nueva realidad de la cultura de finales del siglo XIX. Al igual que en su momento la Secesión Vienesa, el Deustche Werkbund, los Talleres del Estado Vieneses o las teorías de Henri Van de Velde, tampoco las vanguardias arquitectónicas lograrían mover al perro del calor de la estufa. Tampoco ellas conseguirían, pese a su impaciencia, instalar nada verdaderamente nuevo, sino sólo la apariencia de lo nuevo.

El proyecto de las vanguardias históricas también arrastraría otro fatal atentado contra lo moderno, en el que ya habrían incurrido los modernismos finiseculares: su aspiración a la totalidad, su programática conquista de todas las áreas vinculadas al entorno físico y a los objetos del hombre contemporáneo. El afán de dominio, justamente, de todo el espacio habitable que se desprendería de los postulados del "proyecto moderno", guiado ya no por el arte, como en los modernismos del siglo XIX, sino por un paradigma técnico-productivista, representa también un acto de incultura. Como agudamente ha señalado Massimo Cacciari, el proyecto moderno "concibe al espacio como un vacío que hay que llenar, una pura ausencia, una carencia. El espacio es mera potencialidad a disposición del proyecto técnico científico. Precisamente esta concepción del espacio es propia del Arkitect: el espacio es un puro vacío que bay que medir-delimitar, en el que hay que producir las propias formas nuevas". El espacio producto del "proyecto" está, entonces, desprovisto de cualidades y a disposición del arquitecto-técnico. Está también cerrado a las fuerzas de la vida y busca, en última instancia, estar completo.

Contrariamente, para Loos, el espacio habitable es por excelencia un lugar en el que conviven objetos de la más variada y vital procedencia. Es una recolección de cosas y de objetos cambiantes y a menudo heterogéneos, a cuya variedad el marco arquitectónico debe responder y amoldarse. Como ha dicho Cacciari, es un espacio definido por la "copresencia de las cosas y de la arquitectura" " Es un espacio pleno pero a la vez incompleto, abierto a los decisivos aunque quizás pequeños cambios de lo verdaderamente moderno. Como él mismo lo expresa debe ser un "pedazo de la vida sensible de su habitante" ${ }^{10}$. El espacio loosiano, entonces, es un espacio que no cristaliza en un orden definitivo, pero que responde a una unidad, la unidad de lo viviente. Unidad que no puede manifestarse en términos artísticos ni técnicos.

En este punto se pone de manifiesto, de manera muy clara, un paradigma recurrente en el discurso de Loos, el de lo natural. Siguiendo, de hecho, a gran parte de la teoría de arquitectura del siglo XIX, especialmente a la vinculada con el romanticismo, Loos postula que el hombre sólo produce o construye correctamente, cuando lo hace como la Naturaleza crea sus formas y sus seres. Los objetos y las construcciones del hombre deben surgir de las fuerzas culturales que se manifiestan en cada oficio y que cuajan en una tradición, de la misma manera que las formas naturales responden a las fuerzas de la naturaleza. La armonía del entorno humano debe ser una armonía análoga a la de las formas naturales, sin 
11 Ver Adolf Loos: "Arquitectura", op.

cit., pág. 23.

12 Ver Adolf Loos "Directrices para una institución de arte" (1919), op. citt, pág. 110 y "Ornamento y Educación" (1924) op. cit., pág. 214. un orden previo, desplegándose cada una de acuerdo a una determinada necesidad interna y externa, respondiendo cada una a sus propios tiempos y durando lo que dura su materia. El orden del entorno del hombre debe ser un reflejo del orden natural. En el ensayo "Arquitectura" expone claramente esta idea: un entorno natural cualquiera, un lago rodeado de montañas, no se ve alterado por la presencia de la obra del hombre. $\mathrm{Ni}$ una construcción como las casas de los campesinos, ni un hecho técnico como un puente, ni un artefacto como un barco, producen en él ninguna perturbación. Son productos "naturales" de una cultura y entran en sintonía con la obra de la Naturaleza. Sólo la casa proyectada por el arquitecto, según Loos, rompe esta armonía y escapa a la cultura y a la naturaleza ${ }^{11}$.

El espacio loosiano quiere ser como un paisaje del que se excluya cualquier objeto nacido de un proyecto. Como los grandes hechos geográficos, el marco estable de la arquitectura tendría como finalidad darle una armónica acogida a los dispares productos del trabajo humano, cada uno surgido de una irremediable necesidad, siguiendo su propia lógica de constitución y sus ritmos de perduración. Por eso es que el espacio habitable tiene que hacer presente la diversidad de saberes y oficios humanos, en ella encuentra su naturalidad, su sentido estético profundo. En los términos del pensamiento de Loos, es terriblemente mezquino y empobrecedor postular que todos los objetos, los artefactos y los elementos arquitectónicos de un espacio habitable puedan ser creados por una sola persona o siguiendo un único principio en la producción de los distintos objetos.

A lo largo de sus escritos, Loos no sólo se refiere, en este punto, a los intentos de la Secesión vienesa y los otros modernismos de fin de siglo XIX por hacer de la arquitectura una "obra de arte total", sino también a las distintas escuelas alemanas vinculadas al área de la producción, como el Deustsche Werkbund o la Bauhaus. En estos casos no se trataría de hacer artísticos los objetos de uso, sino de buscar un conjunto de normas referidas a la economía de los materiales, la funcionalidad, la simplicidad formal, la corrección técnica, etc., a ser impuestas a toda la producción. También esto es un error para Loos. También estos intentos buscarían el dominio o la colonización de los oficios en nombre de la forma moderna ${ }^{12}$. Pero la forma verdaderamente moderna en los objetos de uso no puede salir de una escuela, de la misma manera que no podía salir de la mente de un artista-arquitecto. Los oficios, que no se preocupan por la cuestión de la forma, que son inocentes y que no intentan inventarla, son los encargados, en su diversidad y multiplicidad, de producir la forma moderna, que en realidad no es más que una actualización de una forma ya trabajada por la tradición.

En este contexto de ideas, la arquitectura es, o debería ser, para Loos, como un oficio más, sin prerrogativas sobre los otros. Como bien ha señalado Aldo Rossi ${ }^{13}$ la lógica del pensamiento loosiano termina por quitarle a la arquitectura su status de profesión, para regenerarla en el fértil suelo de los oficios. Así como el fontanero no interfiere con el ebanista, de la misma manera la arquitectura tampoco debería interferir con los restantes oficios, sino que debería buscar cuidadosamente su propia especificidad y convertirse en oficio. Por esto Loos rechaza el título de arquitecto y prefiere llamarse, simplemente, Baumeister, maestro constructor. El maestro constructor no crea sino que repite o innova mínimamente sobre lo que recibe de la tradición; no tiene una teoría sobre cómo debe ser la casa, pero conoce a su cliente; sabe pensar en términos de espacio pero no sabe dibujar; piensa primero en los materiales y luego en la forma; no puede proponer nuevas formas de vida, solamente aplicar los nuevos avances técnicos donde sean absolutamente necesarios; ama las formas simples pero no profesa la simplicidad; puede incluso colocar ornamentos si su cliente así se lo exige. Trabaja inocentemente, no forma parte de ningún proyecto. Como cualquier oficio. La imbricación de la arquitectura, considerada como oficio, con los otros saberes y prácticas que intervienen en la construcción del entorno humano se vuelve, justamente, natural. Al volverse oficio la arquitectura se naturaliza, se vincula orgánicamente con las restantes fuerzas de la cultura, sus tiempos y sus productos: los automóviles, los zapatos, las estufas y los saleros del hombre moderno. Se restablece de esta forma la armonía de la cultura.

\section{E scritura, materia}

Oficio, naturaleza, cultura: cada una de estas categorías se apoya, para Loos, la una en la otra. La manifestación más característica de esta concordia sería la unidad del entorno físico humano. La idea de proyecto, entendido como programa, como acción transformadora con aspiraciones a la totalidad y, en última instancia, como cauce para el desarrollo de la cultura y la sociedad en un determinado sentido, tal cual lo entendieron las vanguardias de la arquitectura de los años veinte, implicaría para Loos la disolución de esta concordia, rompería su tejido de rela- ciones. Contra esta noción de proyecto general, como ya hemos visto, Loos propone la vuelta al oficio que desagregaría al proyecto, y de la cual su propia obra construida pretende ser una ejemplificación.

Pero también se opone Loos a lo que podríamos llamar las "técnicas del proyecto", a la operación en sí de idear una construcción, tal cual habría cristalizado dentro la profesión de arquitectura y en las artes aplicadas durante el siglo XIX. El rechazo de Loos se dirige principalmente a la que era, y en gran medida aún es, la herramienta central de las formas de proyección desde el Renacimiento: el dibujo. Efectivamente, el dibujo como forma de ideación, como instrumento de estudio y como técnica que supone una cierta autonomía y una destreza propia, conlleva para Loos una distancia demasiado riesgosa entre lo que se representa y lo que se va a construir, entre el presente (el dibujo mismo) y el futuro (el objeto o la obra de arquitectura). El dibujo no permitiría prefigurar adecuadamente las cualidades intrínsecas de una obra de arquitectura o de un objeto, y les impondría, por lo tanto, sus propias cualidades (las del dibujo), que son las cualidades que permiten representar, a las que alcanza en cuanto técnica, pero que no son las de la cosa misma.

La creación de obras de arquitectura tiene que ver básicamente con el dominio del espacio y con el dominio de la materia que genera ese espacio. Ni la materia ni el espacio son aprehensibles o cognoscibles, en su especificidad, en su realidad concreta, por medio del dibujo. El dibujo, según Loos, sólo permite figurar la realidad del espacio y de la materia. Y la figura no es más que un conjunto de líneas y colores sobre un plano, y responde, debido a ello, a otro tipo de problemas que no son los del objeto en sí.

Podría decirse que para Loos el dibujo, por una parte, no es nunca lo suficientemente abstracto como para expresar una idea plenamente, sin que interfiera en uno u otro grado la cuestión de la forma a asumir por esa idea. Pero por otra parte, es demasiado abstracto con relación a la materia con la que se va a construir la obra de arquitectura o se va a producir el objeto. Las cualidades del material son siempre traicionadas por el dibujo, o más radicalmente, el dibujo no logra llegar, por su propia naturaleza, a las cualidades de ese material. Y es sobre estas cualidades sobre las que se debe fundar, para Loos, el proceso de ideación de los objetos de uso y de la arquitectura.

Descartada, entonces, la herramienta central y en gran medida constitutiva del proyecto, cabe preguntarse ¿cómo obrar para producir algo? 
¿Cuáles serían para Loos las herramientas alternativas al dibujo como instancia de ideación previa?

En términos generales, se desprenden de sus artículos dos vías posibles de acción. Ellas serían la palabra escrita y la identificación con la materia. Para Loos, una obra de arquitectura que no puede describirse con palabras no es una obra de arquitectura $\mathrm{a}^{14}$. Los interiores de Ollbrich, por ejemplo, no pueden ser contados, no es posible ponerlos en palabras y que esto dé como resultado un discurso coherente o razonable, porque sólo serían el producto de un dibujo, de un conjunto de intenciones que sólo tienen razón de ser sobre el plano, pero que pierden su sentido en la realidad. Si existe una idea que determina verdaderamente la razón de ser de un objeto o de una construcción, esta construcción o este objeto tienen que poder ser puestos en palabras razonables. La lengua sería un instrumento más fiel para idear los objetos de la cultura que el dibujo, demasiado cercano a las prácticas artísticas. Se trata, sin duda, de una proposición con un fin claramente provocativo, pero que da una clave decisiva para comprender gran parte de la producción de arquitectura de Loos. Su proyecto para el edificio del Chicago Tribune, por ejemplo, no podría comprenderse, ni haber sido pensado, sin esta premisa. Que Loos intentara, en algunos casos, llevarla hasta sus últimas consecuencias, lo demuestra su presentación a la exposición Weissenhof en Stuttgart de 1927, uno de los hitos de la arquitectura moderna dedicado a la vivienda colectiva, a la que envía un proyecto para una colonia agrícola ${ }^{15}$. Exceptuando unos croquis casi infantiles, Loos confía para dar a conocer su proyecto sólo en la palabra, en la minuciosa descripción y explicación de cada una de las partes de su vivienda, de las futuras acciones de sus habitantes y de las consecuencias que este nuevo hábitat tendría para ellos. No suministra ninguna imagen. Sólo palabras. Lo moderno, parece decir su presentación, es sólo lo que puede ser descrito. La segunda herramienta de ideación y de prefiguración que surge del pensamiento de Loos es el conocimiento vital, si cabe la contradicción, de la materia. No se trata, como postulaba la escuela Bauhaus, de conocer la materia a partir de la experimentación, sino de identificarse con ella por medio del trabajo concreto, de saber captar su naturaleza en pos de un fin determinado. Sería éste un proceso, en gran medida, inefable, que sólo un largo contacto con la materia en términos de trabajo directo haría posible. "Piénsese", escribe Loos refiriéndose a un maestro pedrero, "el hombre ha trabajado desde los catorce años, doce horas diarias en el gremio. No es maravilla que vea el mundo diferente del pintor. Cuando una parte de la vida se pasa trabajando sólo en la piedra, se empieza a pensar pétreamente y a ver pétreamente. El hombre desarrolla un ojo pétreo, que vuelve las cosas de piedra. Al hombre se le ha vuelto una mano de piedra, una mano que lo convierte todo en piedra" ${ }^{16}$ Por eso, los oficios, al dividirse según las materias originarias con las que trabajan, terminarían por introducir el orden natural dentro de la cultura y de la sociedad. Al identificarse con el material, quien lo trabaja actúa con él como lo haría la Naturaleza, la forma que produce con él sería análoga a la forma natural. Por eso Loos habla del "soplo de la naturalidad" que evidencian los buenos objetos. Volver a la materia es volver a conectar la cultura con la naturaleza. Según sus propias palabras, "la materia debe ser deificada de nuevo. Los materiales son sustancias misteriosas. Debemos asombrarnos de que algo así exista" ${ }^{17}$.

\section{La vida de la arquitectura}

Existe una doble operación en el pensamiento de Loos. En primera instancia, separar, identificar lo esencialmente distinto, disolver las falsas similitudes. Esto se logra a partir del retorno a la materia, o más precisamente a las múltiples materias. Sobre cada una de éstas se establece una especie de cadena continua de trabajo humano análogo al hacer natural, en las que se fundan los oficios. Este conjunto de corrientes de trabajo no acepta la imposición de ningún proyecto empujado sólo por la voluntad de un grupo de personas, que lo detenga, ni en nombre del arte (como la Secesión), ni en nombre de los valores del pueblo (como el diseño y la arquitectura vernáculos), ni en nombre de lo técnico funcional (como algunas de las vanguardias históricas en el campo de la arquitectura). Esta separación garantiza-ría el orden de la cultura, su unidad y su modernidad.

Pero también puedan señalarse fenómenos comunes a todas estas corrientes de trabajo. La técnica, correctamente comprendida, separada principalmente de la categoría de lo nuevo como se da en el arte y de toda intencionalidad artística, sería uno de estos fenómenos. El ornamento, en su momento, habría sido otro. Estos fenómenos son producto de la cultura en su conjunto y están sujetos a los cambios que se producen en ella. Pueden "morir" en términos culturales, lo que no implica necesariamente que desaparezcan. ¿Qué significa "morir en términos de cultura"? Significa que estos fenómenos dejan de ser constitutivos en la relación del hombre con la realidad exterior. Tomemos el caso del ornamento como aparece explicado a lo largo de toda la producción de Loos y no sólo del ensayo "Ornamento y delito". En principio el hombre habría sentido la imperiosa necesidad de diferenciar sus productos de los de la naturaleza. El ornamento sería justamente esta señal distintiva, que convertiría a un pedazo de madera en un remo. El tatuaje, en última instancia, permitiría al hombre separar su propio cuerpo, y por arrastre su propia conciencia, de la naturaleza. El ornamento permitiría al hombre diferenciar lo artificial de lo natural, lo realizado por él a lo realizado por fuerzas ajenas a él. Al interiorizarse en el hombre esta capacidad de diferenciación, al hacerse connatural a la conciencia del hombre, la necesidad cultural del ornamento habría caducado. Sería insignificante en la relación del hombre con la realidad. Lo que no quiere decir que como práctica vaya a desaparecer nunca completamente o que no pueda tomar otros significados culturales menores. Loos propone en sus trabajos finales una función menor para el ornamento en el mundo contemporáneo, como pequeña felicidad destructiva frente al abrumador trabajo constructivo del mundo moderno. Pero ya no debería llamarse ornamento, sería necesaria otra palabra.

En este punto, la pregunta a la que el pensamiento de Loos conduce resulta inevitable en estos primeros años del siglo veintiuno, así como también resultó inevitable para Manfredo Tafuri en los años setenta: ¿no será la arquitectura también uno de estos fenómenos culturales? ¿No habrá cumplido su ciclo como producto de la cultura moderna, de la misma manera que el ornamento cumplió el suyo en las culturas primitivas? Pensar el fin de la arquitectura en estos términos no implica suponer que dejarán de construirse viviendas, aeropuertos, edificios de oficinas para las corporaciones, malles o gasolineras; ni que dejará de ser necesario un determinado conocimiento para realizar esto, sino que su sentido y su significación dentro de la cultura moderna se ha debilitado. Evidentemente, un edificio ya no media entre el hombre y el universo como pudo haberlo hecho en el Renacimiento y lo edificado ha dejado ese gran instrumento de conocimiento y de mediación con la realidad que fue en algún momento. Curiosamente, hacia esta arquitectura sin proyecto y sin A mayúscula se dirige el pensamiento de Loos.

Alejandro Crispiani Enríquez

Arquitecto Universidad de La Plata, Argentina, 1984. Candidato a Doctor por la Facultad de la Filosofía y Letras de la Universidad de Buenos Aires con la tesis "La trayectoria de la buena forma: del Arte concreto a las teorías del diseño para la periferia". Actualmente es profesor de la Escuela de Arquitectura de la PUC. 